\title{
A comparison of two-dimensional and three- dimensional techniques for determining the kinematic patterns for hindlimb obstacle clearance during sheep locomotion.
}

\section{Camila Cardoso Diogo}

Universidade de Tras-os-Montes e Alto Douro

\section{Bárbara Fonseca}

Universidade de Tras-os-Montes e Alto Douro

Francisca S.M. Almeida

Universidade de Tras-os-Montes e Alto Douro

Luís Maltez da Costa

Universidade de Tras-os-Montes e Alto Douro

José Eduardo Pereira

Universidade de Tras-os-Montes e Alto Douro

\section{Vitor Filipe}

Universidade de Tras-os-Montes e Alto Douro

\section{Pedro Alexandre Couto}

Universidade de Tras-os-Montes e Alto Douro

\section{Stefano Geuna}

Universita degli Studi di Torino

\section{Paulo A. Armada-da-Silva}

Universidade de Lisboa

Ana Colette Maurício ( $\nabla$ acmauricio@icbas.up.pt )

Universidade do Porto Instituto de Ciencias Biomedicas Abel Salazar https://orcid.org/0000-0002-

0018-9363

\section{Artur S.P. Varejão}

Universidade de Tras-os-Montes e Alto Douro

\section{Methodology article}

Keywords: Sheep, 2D and 3D gait analysis, Locomotion, Obstacle avoidance, Peripheral nerve injury

Posted Date: June 8th, 2020 
DOI: https://doi.org/10.21203/rs.3.rs-27991/v1

License: (c) (1) This work is licensed under a Creative Commons Attribution 4.0 International License. Read Full License 


\section{Abstract}

Background: Analysis of locomotion is often used as a measure for impairment and recovery following experimental peripheral nerve injury. Compared to rodents, sheep offer several attractive features as an experimental model for studying peripheral nerve regeneration. There are no studies on locomotion outcomes after peripheral nerve injury and repair in the sheep model. In the present study, we performed and compared two-dimensional (2D) and, for the first time, three-dimensional (3D) hindlimb kinematics during obstacle avoidance in the ovine model. This study aimed to obtain kinematic data to serve as a template for an objective assessment of the ankle joint motion in future studies of common peroneal nerve (CP) injury and repair in the ovine model.

Results: The strategy used by the sheep to bring the hindlimb over a moderately high obstacle, set to $10 \%$ of its hindlimb length, was the pronounced knee, ankle and metatarsophalangeal flexion when approaching and clearing the obstacle. Despite the overall time course kinematic patterns about the hip, knee, ankle, and metatarsophalangeal were identical, we found significant differences between values of the $2 \mathrm{D}$ and $3 \mathrm{D}$ joint angular motion.

Conclusions: Our results show that the most apparent changes that occurred during the gait cycle were for the ankle and metatarsophalangeal joints, whereas the hip and knee joints were much less affected. Data and techniques described here are likely to be useful for an objective assessment of altered gait after CP injury and repair in an ovine model.

\section{Background}

Because of their spread distribution throughout the body, peripheral nerves are particularly subject to traumatic injuries in animals and humans [1-3]. Given that most experimental studies on microsurgical repair have been performed on major hindlimb nerves, animal gait analysis is often used as a measure for impairment and recovery. Over the years, several gait kinematics have been developed to analyse locomotor deficits following injury in rodents, particularly in the rat sciatic nerve model $[4,5]$.

Although rodents have been intensively used for research, the rate of successful translation from rodent models to clinical applications is low and are driving scientists to pursue different animal models [6]. Sheep offer several attractive features as model organisms for studying peripheral nerve injury and repair. First, nerves in sheep and in humans have similar size and regeneration behaviour $[7,8]$. Second, they are attractive models for biomedical and basic biological research because of availability and they can be kept in natural groups with very basic housing demands. Third, they are placid animals and can be readily trained for gait analysis. Fourth, the larger size of the sheep facilitates more precise and potentially more accurate measurement of joint kinematics. Fifth, due to its relatively large size, sheep provide sufficient nerve tissue samples for other histological and molecular biological analysis. Finally, in comparison to other large mammals like primates, dogs and cats, sheep are better accepted by society as a research animal and also represent a less expensive alternative [9]. 
Animal studies using the sheep as kinematic model are scarce in the literature and only in the field of orthopaedics and spinal cord injury research [10-13]. Recently, our lab used a two-dimensional (2D) model to analyse the hindlimb kinematics in clinically healthy sheep during overground and obstacle clearance during locomotion [14]. We were able to quantify the compensatory strategies that take place at each joint in response to obstacle clearance during locomotion in the ovine model. A considerable advantage of using a 2D kinematic approach is the simplicity of the procedure, which requires only one camera to record the movement. However, it seems reasonable to assume that the hip, knee, ankle and metatarsophalangeal joints may translate out of a parasagittal plane under some conditions, such as obstacle clearance.

In the light of reported literature, no comparisons have been made between 2D and three-dimensional (3D) hindlimb kinematics during obstacle clearance in the ovine model. The purpose of this study was to compare hindlimb kinematics using 2D and 3D approaches in clinically normal sheep during obstacle negotiation and create a template for an objective assessment of the ankle joint motion following common peroneal nerve (CP) injury and repair in the ovine model.

\section{Results}

To ensure a reliable comparison between the 2D and 3D sagittal plane kinematics of the hindlimb joints, a total of 40 gait cycles were obtained during obstacle clearance from 4 clinically healthy sheep.

\section{Spatio-temporal Measurements}

Mean ( \pm S.D.) values obtained for the whole group were $1.36 \pm 0.10 \mathrm{~m} / \mathrm{s}$ for walking speed, $693 \pm 45 \mathrm{~ms}$ for gait cycle duration, $416 \pm 57 \mathrm{~ms}$ for stance duration, $277 \pm 25 \mathrm{~ms}$ for swing duration, $94 \pm 5.0 \mathrm{~cm}$ for stride length and $10.1 \pm 0.8 \mathrm{~cm}$ for MVD during obstacle clearance.

\section{Angular Kinematic Parameters}

The general shapes of the 2D and 3D joint kinematic patterns for the hip, knee, ankle and MTP were very similar (Fig. 4). The average toe-off (TO) time was calculated to be $60 \%$ into the step cycle. Despite similar 2D and 3D overall joint patterns, for MTP, minimal angle during stance (STANCEmin) $(P<0.01$; Table 1) and the angle at TO were both found to be significantly increased (i.e., less flexion) when measured in 3D $(P<0.05$; Table 1A). For the ankle, the maximal angle during stance (STANCEmax) was decreased when recorded in 3D $(P<0.05$; Table 1$)$. No other differences in hindlimb kinematics between the two setups existed. 
Table 1

Joint angle data.

\begin{tabular}{|c|c|c|c|c|c|c|c|c|}
\hline & \multicolumn{2}{|c|}{ MTP (degrees) } & \multicolumn{2}{|c|}{ Ankle (degrees) } & \multicolumn{2}{|c|}{ Knee (degrees) } & \multicolumn{2}{|c|}{ Hip (degrees) } \\
\hline & $2 D$ & $3 D$ & $2 \mathrm{D}$ & $3 D$ & $2 \mathrm{D}$ & $3 \mathrm{D}$ & $2 \mathrm{D}$ & $3 D$ \\
\hline IC & $\begin{array}{l}176 \pm \\
3.3\end{array}$ & $\begin{array}{l}175 \pm \\
2.7\end{array}$ & $\begin{array}{l}145 \pm \\
7.8\end{array}$ & $\begin{array}{l}147 \pm \\
4.8\end{array}$ & $\begin{array}{l}137 \pm \\
12.2\end{array}$ & $\begin{array}{l}143 \pm \\
6.7\end{array}$ & $93 \pm 8.6$ & $\begin{array}{l}96 \pm \\
3.4\end{array}$ \\
\hline STANCEmin & $\begin{array}{l}151 \pm \\
2.2\end{array}$ & $\begin{array}{l}162 \pm \\
2.2^{\star \star}\end{array}$ & $\begin{array}{l}127 \pm \\
4.9\end{array}$ & $\begin{array}{l}130 \pm \\
3.1\end{array}$ & $\begin{array}{l}110 \pm \\
9.2\end{array}$ & $\begin{array}{l}107 \pm \\
4.9\end{array}$ & $93 \pm 8.9$ & $\begin{array}{l}96 \pm \\
3.4\end{array}$ \\
\hline STANCEmax & $\begin{array}{l}177 \pm \\
3.0\end{array}$ & $\begin{array}{l}178 \pm \\
2.0\end{array}$ & $\begin{array}{l}157 \pm \\
2.4\end{array}$ & $\begin{array}{l}151 \pm \\
1.2^{\star}\end{array}$ & $\begin{array}{l}137 \pm \\
12.2\end{array}$ & $\begin{array}{l}143 \pm \\
6.2\end{array}$ & $\begin{array}{l}114 \pm \\
9.7\end{array}$ & $\begin{array}{l}111 \pm \\
7.4\end{array}$ \\
\hline TO & $\begin{array}{l}163 \pm \\
4.9\end{array}$ & $\begin{array}{l}177 \pm \\
1.4^{\star}\end{array}$ & $\begin{array}{l}151 \pm \\
3.7\end{array}$ & $\begin{array}{l}149 \pm \\
1.7\end{array}$ & $\begin{array}{l}114 \pm \\
6.3\end{array}$ & $\begin{array}{l}109 \pm \\
6.7\end{array}$ & $\begin{array}{l}114 \pm \\
10.2\end{array}$ & $\begin{array}{l}111 \pm \\
7.4\end{array}$ \\
\hline SWINGmin & $\begin{array}{l}138 \pm \\
16\end{array}$ & $\begin{array}{l}141 \pm \\
2.6\end{array}$ & $\begin{array}{l}96 \pm \\
4.6\end{array}$ & $\begin{array}{l}101 \pm \\
4.4\end{array}$ & $86 \pm 9.7$ & $\begin{array}{l}94 \pm \\
4.4\end{array}$ & $91 \pm 6.9$ & $\begin{array}{l}94 \pm \\
4.4\end{array}$ \\
\hline SWINGmax & $\begin{array}{l}182 \pm \\
2.3\end{array}$ & $\begin{array}{l}179 \pm \\
0.3\end{array}$ & $\begin{array}{l}151 \pm \\
3.7\end{array}$ & $\begin{array}{l}150 \pm \\
1.1\end{array}$ & $\begin{array}{l}134 \pm \\
11.1\end{array}$ & $\begin{array}{l}141 \pm \\
6.4\end{array}$ & $\begin{array}{l}114 \pm \\
10.4\end{array}$ & $\begin{array}{l}111 \pm \\
7.2\end{array}$ \\
\hline
\end{tabular}

Intra-trial ICC values for joint kinematics data are displayed in Table 2. As could be expected, ICC values ranged between good (values between 0.75 to 0.90 ) to excellent (values above 0.90 ) for most joint kinematics data. However, a few measurements displayed low reliability. The latter mainly pertained to joint angles measured from the distal joints of the hindlimbs, namely the MTP and the ankle (Table 2).

Table 2

Intra-class correlation values for inter-step joint angle data.

\begin{tabular}{|lllllllll|}
\hline & \multicolumn{2}{l}{ MTP (degrees) } & \multicolumn{2}{l}{ Ankle (degrees) } & \multicolumn{2}{c}{ Knee (degrees) } & \multicolumn{2}{l|}{ Hip (degrees) } \\
\hline & 2D & 3D & 2D & 3D & 2D & 3D & 2D & 3D \\
\hline IC & 0.447 & 0.840 & 0.976 & 0.838 & 0.907 & 0.936 & 0.989 & 0.977 \\
\hline STANCEmin & 0.703 & 0.691 & 0.961 & 0.827 & 0.922 & 0.948 & 0.991 & 0.977 \\
\hline STANCEmax & 0.561 & 0.900 & 0.853 & 0.594 & 0.907 & 0.921 & 0.995 & 0.960 \\
\hline TO & 0.949 & 0.352 & 0.891 & 0.187 & 0.926 & 0.949 & 0.993 & 0.956 \\
\hline SWINGmin & 0.968 & -0.176 & 0.877 & 0.807 & 0.808 & 0.603 & 0.986 & 0.966 \\
\hline SWINGmax & 0.127 & 0.503 & 0.891 & 0.306 & 0.901 & 0.959 & 0.992 & 0.954 \\
\hline
\end{tabular}

\section{Discussion}


The purpose of this study was to compare joint hindlimb kinematics in normal sheep during obstacle clearance calculated by a 2D and a 3D method. The 2D and 3D calculations were based on kinematic data from the same experiments to ensure that the comparison was reliable. These assessments provide novel information on how each joint contributes to obstacle clearance during overground locomotion in the ovine model, while also demonstrating the good to excellent reliability of amplitudes and timing of joint trajectories when recorded in a single session. In order to reduce the flocking behaviour, 2 weeks before data collection, sheep were trained to walk over the walkway at a comfortable speed, led by the same handler using a halter. This improved the accuracy of the kinematic gait parameters and minimized the number of animals used. [15].

In future studies of surgical transection and repair of peripheral nerves, we will selectively choose the common peroneal nerve (CP) in the ovine model. Sheep CP is a medium nerve with a diameter similar to frequently injured human nerves, allowing for much longer nerve gaps when compared to rats. The CP innervates only muscles of dorsiflexion, namely, the cranial tibial and peroneus tertius, and no antagonistic muscle groups. An important consequence of an experimental CP injury is the loss of ankle dorsiflexor activity. Therefore, this new biomechanical model will allow an objective assessment of ankle locomotor mechanics and the compensatory strategies for future nerve regeneration studies.

In this model, a $6 \mathrm{~cm}$ high obstacle was placed in the middle of the walkway and all animals showed an adequate ankle dorsiflexion, allowing the necessary hoof-clearance, while maintaining forward progression. Choosing the correct height for the obstacle, reflecting $10 \%$ of the animals' hindlimb length, was essential for a safe and successful obstacle-crossing, as previously described. $[15,16]$.

The results of the $2 \mathrm{D}$ and $3 \mathrm{D}$ kinematic data showed that the general shapes of joint angular motion were very similar and only few statistically significant differences in angle values at selected points of the gait cycle existed between the two setups. Differences between 2D and 3D joint kinematics were only found at the MTP and the ankle, whereas the magnitudes of the hip and knee flexion-extension angles were the same, regardless of being recorded in $2 \mathrm{D}$ or $3 \mathrm{D}$.

Although our results support the use of the simpler 2D method, as most of the joint angles were similarly measured in 2D and 3D, there were some consistent differences between the two motion analysis methods. Previous studies also report differences in rat toe and ankle flexion-extension joint position during level walking between 2D and 3D biomechanical analysis, which agrees with the present results [17]. The fact that 3D-measured MTP and ankle angles might subtly but consistently differ from 2D could be relevant for applications in peripheral nerve research employing the sheep model and the CP nerve, as injury of this nerve specifically affects innervation of the skeletal muscles crossing those joints. Further studies comparing 2D and 3D sheep hindlimb motion using animals recovering from CP injury are warranted.

The high degree of stability of hindlimb flexion-extension in our biomechanical model was also demonstrated by the good to excellent inter-step reliability observed for a large majority of gait measurements in this study. However, poor reliability was found for a limited number of MTP and ankle 
angle measurements. At this point, it is uncertain whether such low ICC values reflect normal variation in joint kinematics during obstacle negotiation or is related to study design, in particular the limited number of animals.

It is important to acknowledge several limitations of the data collected for this study.

First, incorrect placement of markers with respect to the anatomy is a known source of error in the joint kinematic outputs [18]. However, since one investigator applied the markers over palpable bony landmarks on all study animals, it is expected that these errors would be minimized and be similar across all trials.

Second, displacement of the skin surface relative to knee joint is the principle source of error when estimating hindlimb joint kinematics in quadrupeds, due to a more extensive skin attachment from the proximal hindlimb to the lateral torso when compared to humans [19]. The 3D biomechanical knee model was made possible by using radiography and applying the anatomical findings to biomechanical principles. The knee joint should operate aligned with the plane defined by the greater trochanter, the knee and the lateral malleolus. We may consider the knee as a hinge joint with 1 degree of freedom, moving from extension into full flexion along the sagittal plane [20]. Third, we did not collect EMG recordings in this study. As a result, we cannot compare the observed changes in joint motion with the recruitment of the different muscles of the hindlimb.

\section{Conclusions}

In summary, the present study established fundamental 3D kinematic characteristics of ankle joint motion and the compensatory strategies that take place at each joint of the hindlimb in response to obstacle clearance in the ovine model. The kinematic results presented here should provide direction for future studies. It is expected that this in vivo 3D hindlimb model will prove useful to assess locomotion of sheep following CP injury and repair. We also anticipate that this model will serve as an effective tool to compare gait pathologic conditions and improve our knowledge on kinematic features associated with other experimental neurological conditions.

\section{Methods}

\section{Ovine model}

This investigation was approved by the Institutional Animal Care and Use Committee of the University of Trás-os-Montes e Alto Douro (IACUC Approval No. 6/2015). All procedures were performed with the approval of the Portuguese Veterinary Authorities, in accordance with the EU Directive 2010/63/EU for animal experiments. The experimental sheep were female Portuguese Churra-da-Terra Quente breed (age: $\approx 2$ years; weight: $\approx 40 \mathrm{~kg}$ ) belonging to a breeding colony based at University of Trás-os-Montes e Alto Douro. Upon delivery, all animals were examined by a veterinarian and deemed non-pregnant and free of disease. The sheep were judged to be healthy on account of results of complete physical, orthopaedic 
and neurologic examinations. The animals were fed a diet of hay supplemented with concentrate according to their requirements and had free access to fresh water. The present experimental design did not induce any pain or discomfort to the included ovine, and by the end of the studied timepoint, all the animals returned to their normal activities without any disturbance nor pathology.

\section{Training Procedure}

The general procedure for training was similar to that in previous work [14]. Two weeks before the collection of kinematic data, sheep were trained daily for 30 minutes to walk over ground at a natural speed on a $2 \mathrm{~m}$ by $0.6 \mathrm{~m}$ walkway and to step over a rectangular obstacle $(6 \mathrm{~cm}$ high $\times 6 \mathrm{~cm}$ wide).

\section{Kinematic Recording}

Hemispherical markers with a diameter of $2 \mathrm{~cm}$ were placed on the skin over six anatomic landmarks on the lateral side of the left hindlimb: the iliac crest, the greater trochanter, the knee joint, the lateral malleolus, the distal end of the metatarsal bone and distal end of the middle phalanx, as in the previous study [14].

Three CMOS cameras (PhotonFocus MV-D640C, Lachen, Switzerland) were strategically placed around the left hindlimb to minimize marker occlusion, maximize resolution and to improve the accuracy of the $3 \mathrm{D}$ reconstruction process. The camera in the middle, which was placed perpendicular to the direction of the movement, was used for 2D analysis. Kinematic data were collected at a sampling rate of $144 \mathrm{~Hz}$. The cameras' field of view was calibrated to cover 2 meters in length of the walkway and allowed the recording of a complete gait cycle (Fig. 1). The images were acquired using the software Video Savant 4 (IO Industries Inc, Ontario, Canada). The colour image had a resolution of $640 \times 480$ pixels. The camera calibration and the 3D reconstruction process were similar to a previously described procedure [21].

During each trial, sheep walked at a natural speed over the walkway, led by a handler using a halter. The present work limited walking velocity to between 1.1-1.5 m/s [14]. A total of ten trials for each sheep were analysed.

Joint flexion-extension angles were measured at the flexor side for hip, knee, ankle and metatarsophalangeal (MTP) joints. The 2D approach was described in detail previously [14, 22]. Briefly, the knee position was computed indirectly by superimposing two circles (centred on hip and ankle pivots) with a radius of the femur and tibia length, respectively. The knee position was determined as the intersection of the two circles. For the 3D biomechanical model, we applied a slightly different approach, as previously described (Fig. 2) [24]. The two circles were replaced by two spheres centred on the greater trochanter and the lateral malleolus with a radius equal to the length of the femur and tibia and segments, respectively. We presumed that the true position of the knee marker would lie on the plane defined by the greater trochanter, the knee and the lateral malleolus markers. 


\section{Numerical Analysis}

The general procedure for numerical analysis was similar to our recent paper [14].

The marker-based angular kinematic curves were filtered using a fourth-order Butterworth filter (cut-off frequency at $10 \mathrm{~Hz}$ ). For each stride, the duration of the stance and swing phases was normalized. Cubic spline interpolation was applied to the original data regarding the angular position of the pelvis, hip, knee, ankle, and MTP joints to obtain 101 samples per gait cycle regardless of their duration. We recorded the maximal and minimal joint flexion-extension angles during both the stance and swing phases of the gait, as well as the angles at the point of initial hoof-ground contact (IC, start of the stance phase) and immediately when the hoof is lifted from the ground (TO, start of the swing phase). This numerical analyse was performed using Matlab computational software (The MathWorks Inc., Natick, MA, USA).

In addition, the following gait parameters were included: gait cycle duration, stance duration, swing duration, stride length and the maximal vertical displacement (MVD). Stride length was defined by the distance between the middle phalanx markers of the hindlimb in two consecutive steps. Maximal vertical displacement was quantified by measuring the maximum height reached by the middle phalanx marker when stepping over the obstacle (Fig. 3).

\section{Statistical analysis}

Differences in joint kinematics data collected in 2D and 3D were tested using paired Student's t-test. Mean standard deviation (SD) values for all the measured variables are reported. The two-way mixed model intra-class correlation coefficient (ICC) for absolute agreement was calculated as a preliminary measurement of intra-trial, inter-step reliability of joint kinematics data. The statistical significance was set at the level of $P<0.05$. All statistical tests were performed using SPSS v. 22 software (Statistical Package for the Social Sciences Inc., Chicago, USA).

\section{Abbreviations}

2D: Two-dimensional; 3D: Three-dimensional; CP: Common peroneal nerve; IC: Initial contact; ICC: intraclass correlation coefficient; MVD: Maximal vertical displacement; MTP: Metatarsophalangeal; SD: Standard deviation; STANCEmax: maximal angle during stance; STANCEmin: minimal angle during stance; SWINGmax: maximal angle during swing; SWINGmin: minimal angle during swing; TO: Toe-off

\section{Declarations}

\section{Ethics approval and consent to participate}

This investigation was approved by the Institutional Animal Care and Use Committee of the University of Trás-os-Montes e Alto Douro (IACUC Approval No. 6/2015). All procedures were performed with the approval of the Portuguese Veterinary Authorities, in accordance with the EU Directive 2010/63/EU for 
animal experiments. All the authors consented to participate in the manuscript and in the experimental research involved and described in the manuscript.

\section{Consent for publication}

Not applicable

\section{Availability of data and materials}

All data generated or analysed during this study are included in this published article. Any other information is available from the corresponding author on reasonable request.

\section{Competing interests}

The authors declare that the research was conducted in the absence of any commercial or financial relationships that could be construed as a potential conflict of interest.

\section{Funding}

This research was supported by COMPETE 2020, from ANI - Projetos ID\&T Empresas em Copromoção, by the project "Print-on-Organs - Engineering bioinks and processes for direct printing on organs" with the reference POCI-01-0247-FEDER-033877, and by the project "Bone2Move - Development of "in vivo" experimental techniques and modelling methodologies for the evaluation of 4D scaffolds for bone defect in sheep model: an integrative research approach" with the reference POCI-01-0145-FEDER-031146.

\section{Authors' contributions}

CCD, BF, FSMA, LMC, JEP, and PAS had a contribution to the acquisition, analysis of data; SG, ACM, and ASPV had a contribution in conception, design of the work, interpretation of data and substantively revised the work, CCB and ASPV have drafted the work, VF, PAC were involved in the creation of new software and acquisition, analysis of data.

\section{Acknowledgments}

The authors would like to gratefully acknowledge Dr. Marília de Albuquerque Bonelli for English language revision.

\section{References}

1. Mohanna PN, Young RC, Wiberg M, Terenghi G. A composite poly-hydroxybutyrate-glial growth factor conduit for long nerve gap repairs. J 2003;203:553-65.

2. Forterre F, Tomek A, Rytz U, Brunnberg L, Jaggy A, Spreng D. latrogenic sciatic nerve injury in eighteen dogs and nine cats (1997-2006). Vet Surg. 2007; 36:464-71. 
3. Siemionow M, Brzezicki G. Current techniques and concepts in peripheral nerve repair. Int Rev 2009;87:141-72.

4. Costa LM, Simões MJ, Maurício AC, Varejão AS. Chapter 7: Methods and protocols in peripheral nerve regeneration experimental research: part IV-kinematic gait analysis to quantify peripheral nerve regeneration in the rat. Int Rev 2009;87:127-39.

5. Vela, F.J., Martínez-Chacón, G., Ballestín, A., Campos, J.L., Sánchez-Margallo, F.M., Abellán E., 2020. Animal models used to study direct peripheral nerve repair: a systematic review. Neural Regeneration Research 15, 491-502.

6. Angius D, Wang H, Spinner RJ, Gutierrez-Cotto Y, Yaszemski MJ, Widebank, AJ. A systematic review of animal models used to study nerve regeneration in tissue engineered scaffolds. Biomaterials 2012;33:8034-9.

7. Glasby MA, Mountain RE, Murray JA. Repair of the facial nerve using freeze-thawed muscle autografts: a surgical model in the sheep. Arch Otolaryngol Head Neck Surg. 1993;119:461-5.

8. Fullarton AC, Myles LM, Lenihan DV, Hems, TE, Glasby MA. Obstetric brachial plexus palsy: a comparison of the degree of recovery after repair of a $\mathrm{C} 6$ ventral root avulsion in newborn and adult sheep. Br J Plast Surg. 2001;54:697-704.

9. Ozturk C, Uygur S, Lukaszuk M, 2016. Sheep as a large animal model for nerve regeneration studies. In: Siemionow MZ. Plastic and Reconstructive Surgery. Chicago: Springer; 2016. 506-11.

10. Beveridge JE, Shrive NG, Frank CB. Meniscectomy causes significant in vivo kinematic changes and mechanically induced focal chondral lesions in a sheep model. J Orthop Res. 2011;29:1397-405.

11. Frank CB, Beveridge JE, Huebner KD, Heard BJ, Tapper JE, O’Brien EJ, et al. Complete ACL/MCL deficiency induces variable degrees of instability in sheep with specific kinematic abnormalities correlating with degrees of early osteoarthritis. J Orthop Res. 2012;30:384-92.

12. Valentin S, Essigbeck A, Wolfram I, Licka T. Kinematic parameters of sheep walking on a treadmill. Vet J. 2014;202:657-8.

13. Wilson S, Abode-lyamah KO, Miller JW, Reddy G, Safayi S, Fredericks DC, et al. An ovine model of spinal cord injury. J Spinal Cord Med. 2017;40:346-60.

14. Costa D, Diogo CC, Costa LMD, Pereira JE, Filipe V, Couto PA, et al. Kinematic patterns for hindlimb obstacle avoidance during sheep locomotion. Neurological Res. 2018;40:963-71.

15. Hargreaves AL, Hutson GD. Handling systems for sheep. Livest Prod 1997;49:121-38.

16. Niang AE, McFadyen BJ. Effects of physical activity level on unobstructed and obstructed walking in young male adults. Gait Posture 2005;22:75-81.

17. João F, Amado S, Veloso A, Armada-da-Silva P, Maurício AC. Anatomical reference frame versus planar analysis: Implications for the kinematics of the rat hindlimb during Locomotion. Rev Neurosci. 2010;21:469-85.

18. McGinley JL, Baker R, Wolfe R, Morris ME. The reliability of three-dimensional kinematic gait measurements: a systematic review. Gait Posture 2009;29:360-9. 
19. Muir GD, Webb AA. Mini-review: assessment of behavioural recovery following spinal cord injury in rats. Eur J 2000;12:3079-86.

20. Ekeberg O, Pearson K. Computer simulation of stepping in the hind legs of the cat: an examination of mechanisms regulating the stance-to-swing transition. J 2005;94:4256-68.

21. Diogo CC, Costa LMD, Pereira JE, Filipe V, Couto PA, Magalhães LG et al. Dynamic feet distance: A new functional assessment during treadmill locomotion in normal and thoracic spinal cord injured rats. Behav Brain Res. 2017;335:132-5.

22. Filipe VM, Pereira JE, Costa LM, Magalhães LG, Maurício AC, Couto PA, et al. Effect of skin movement on the analysis of hindlimb kinematics during treadmill locomotion in rats. J Neurosci Methods 2006;153:55-61.

23. Couto PA, Filipe VM, Magalhães LG, Pereira JE, Costa LM. Melo-Pinto P, et al. A comparison of two dimensional and three-dimensional techniques for the determination of hindlimb kinematics during treadmill locomotion in rats following spinal cord injury. J Neurosci Methods 2008;173:193-200.

\section{Figures}

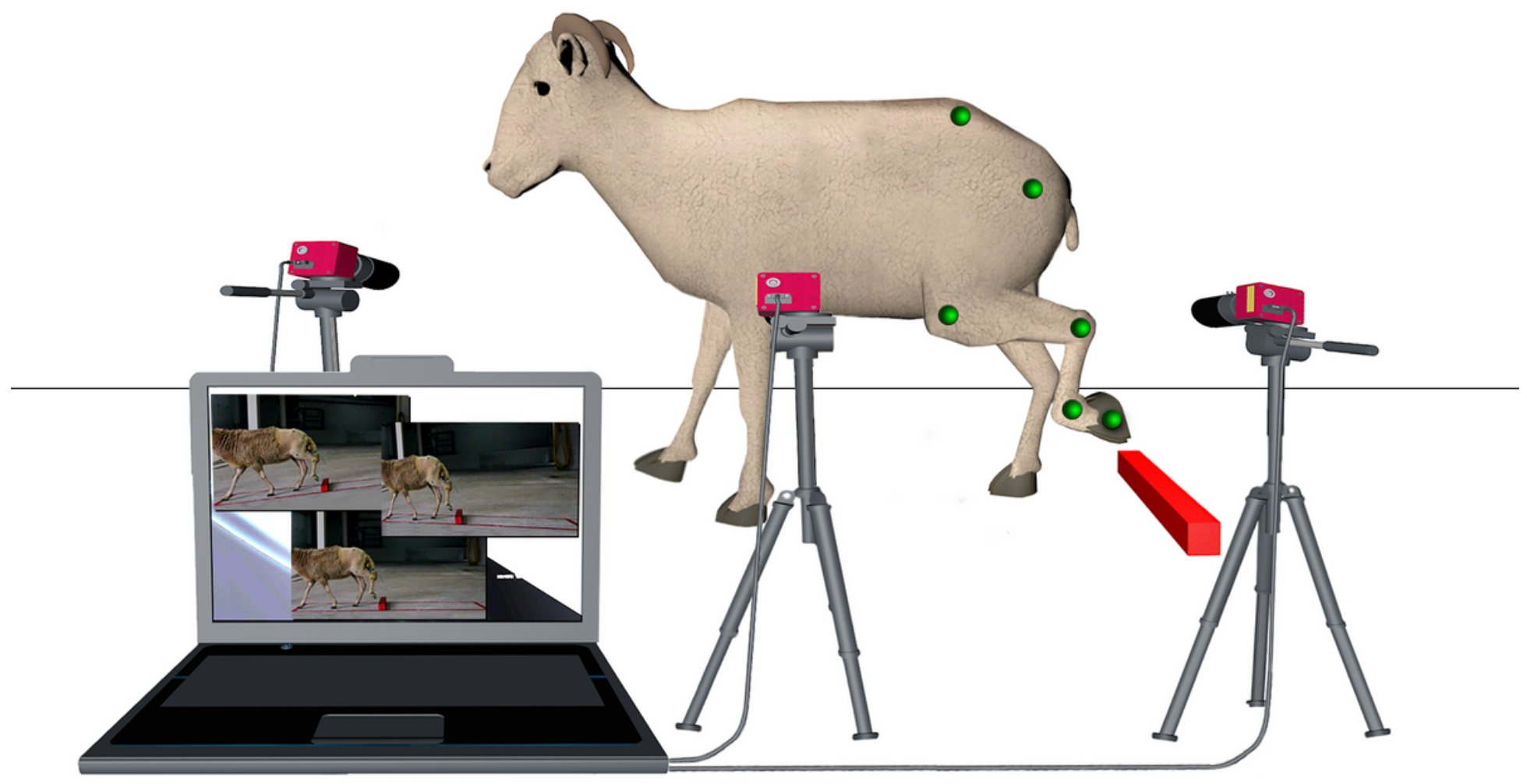

Figure 1

Depiction of the gait laboratory set up for simultaneous 2D and 3D gait analysis of hindlimb obstacle clearance. Three high-speed digital cameras were strategically positioned around the left hindlimb to minimize marker occlusion and maximize resolution. Hemispherical markers with a diameter of $2 \mathrm{~cm}$ 
were placed at various points on the lateral side of left hindlimb and the angle measurements taken at the flexor side of each joint.



Figure 2

Three-dimensional estimation of the knee marker. The knee position was computed indirectly by superimposing two spheres centred on the 3D positions of the greater trochanter (red) and the lateral malleolus (blue) and with a radius equal to the length of the femur and tibia segments, respectively. It 
was presumed that the true position of the knee marker would lie on the plane defined by the greater trochanter, the knee and the lateral malleolus markers. From the intersection of this plane with the circumference (green) resulting from the intersection of the two spheres, we obtained two points. We chose the one that was consistent with the direction of movement. (For interpretation of the references to colour in this figure legend, the reader is referred to the web version of the article.)

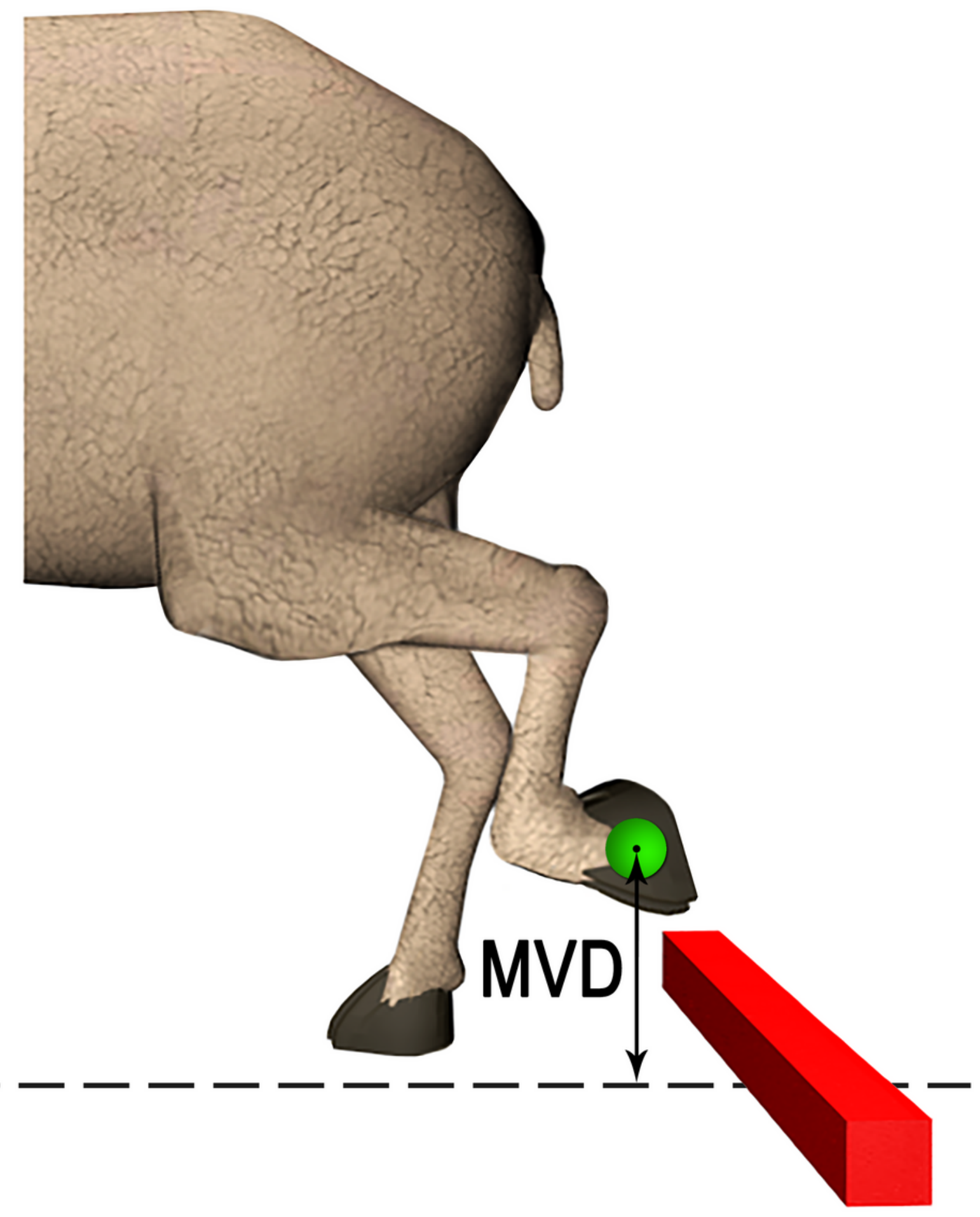

Figure 3 
Schematic representation of the sheep hindlimb showing the maximal vertical displacement (MVD) convention considered in the present study. MVD was quantified by measuring the maximum height the middle phalanx marker reached during the obstacle encounter.

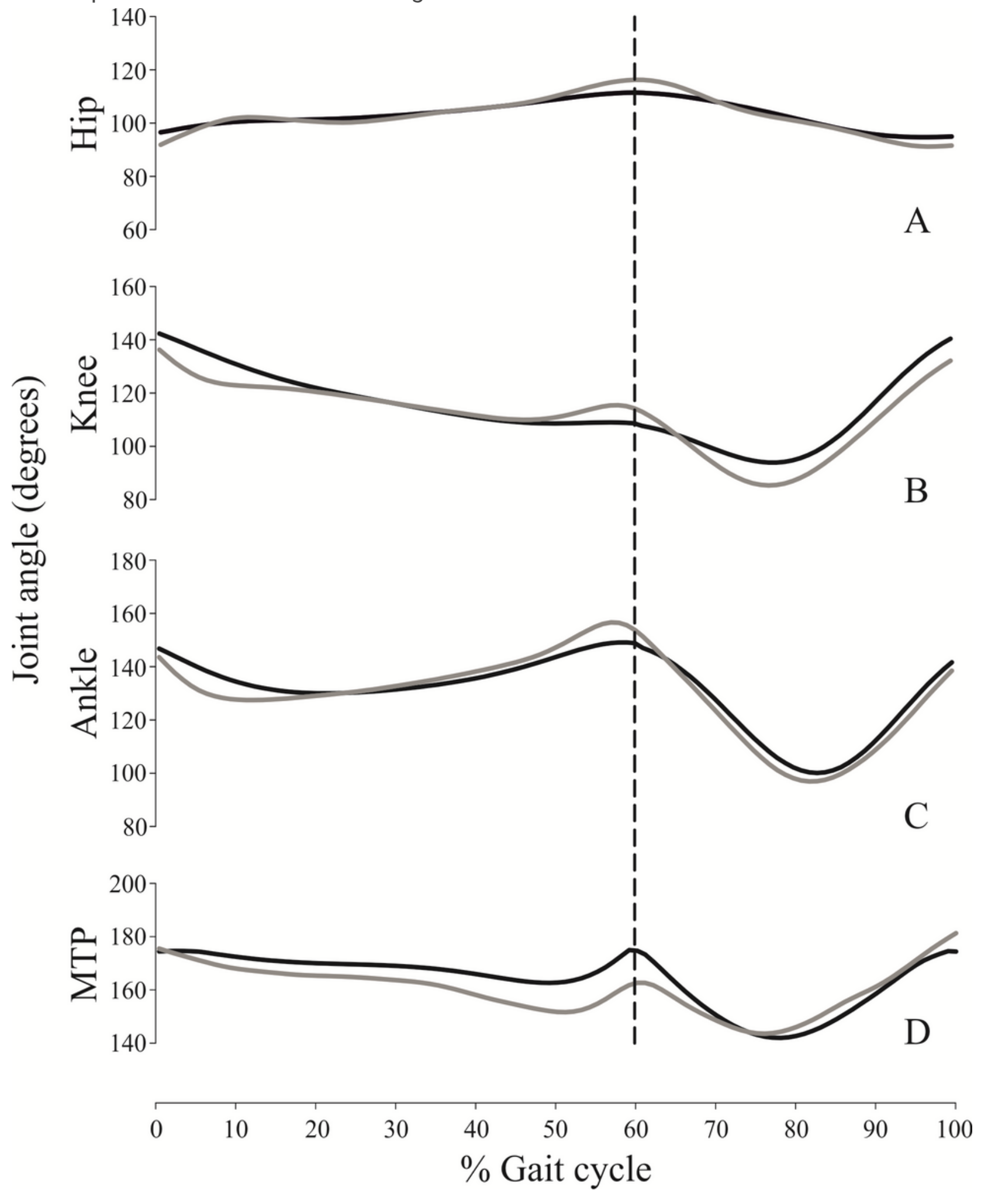

Figure 4

Mean values for the joint angular positions of the hip (A), knee (B), ankle (C) and metatarsophalangeal joint (MTP) (D), using a 2D (grey trace) and a 3D (black trace) approach. Stance and swing phases were 
normalized. The stance duration was set at $60 \%$ of the gait cycle duration. The vertical dashed line corresponds to the stance-swing transition. 